MATEC Web of Conferences 24,06003 (2015)

DOI: $10.1051 /$ matecconf/ 20152406003

(c) Owned by the authors, published by EDP Sciences, 2015

\title{
Dynamic properties comparisons between experimental measurements and nondeterministic numerical models of viscoelastic sandwich beams
}

\author{
W. N. Felippe Filho ${ }^{1, a}$, F. Barbosa ${ }^{1}$ \\ ${ }^{1}$ Federal University of Juiz de Fora, Graduation Program in Computational Modeling, Juiz de Fora, Brazil
}

\begin{abstract}
In order to design viscoelastic sandwich structures used as passive damping treatment, many aspects should be considered. In all methods available in the literature to model Viscoelastic Materials (VEM) a crucial step is the determination of the complex modulus, usually obtained by curve fitting experimental results. Considering that dispersions are inherent to experimental tests and also those small variations in the fitted parameters lead to considerable changes on the dynamic behavior of VEMs hence a nondeterministic model seems to be more suitable than the usual deterministic ones. In that way, starting from dynamic properties of a VEM, a nondeterministic numerical model, which takes into account incertitudes in the VEM curve fitting procedure, is proposed. This model was used to evaluate the behavior of sandwich structures, showing the advantages and disadvantages of the presented methodology, comparing damping ratios and natural frequencies results of experimental tests with the ones extracted from the proposed nondeterministic numerical GHM based model, in order to establish a method to support viscoelastic sandwich beams design.
\end{abstract}

\section{Introduction}

Aiming the reduction of structural vibrations, several techniques were developed to increase structural damping. Among these techniques, the passive control with viscoelastic materials has shown reasonable efficiency. These materials have low bearing properties with high dissipative capacity when subjected to cyclic deformations. That is the main reason that justifies the wide application of VEM in sandwich layers with stiff elastic materials working as a passive control system.

This type of control systems have experienced a growth in practical applications also due to some benefits related to cost-effectiveness [1-4].

Computational modeling of VEM materials may be performed in frequency domain or in time domain. Due to the frequency dependence of mechanical properties of VEM, time domain based models are not as numerous as frequency domain methods. In spite of that, In view of the facilities that time domain methods may directly provide, such as the maximum displacement range in a structural model analysis, many researchers have been developing numerical methods to simulate the dynamical response of VEM in time domain.

Among these time-domain based methods for VEM, those that introduce extra dissipation coordinates or internal variables in order to apply the Finite Element Method (FEM), has been applied in several situations such as the ones presented by [5-8]. In this context, It is possible to observe that Golla-Hughes-McTavish (GHM)

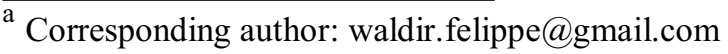

method $[9,10]$ is frequently chosen in order to simulate the dynamic response of VEM.

In a previous work [11], it was presented an assessment including experimental and numerical analysis of VEMs. Viscoelastic materials, applied as the core of sandwich beams, were experimentally tested and GHM method was able to fairly simulate the dynamic behavior of these assayed beams. In this paper, GHM method is validated and it was observed the importance of the curve fitting procedure in order to achieve good results in the computational simulation.

In another previous work, [12], the influence of the fitted parameters in the computational model results was carried out, showing that small variations in these parameters lead to considerable changes on the dynamic behavior of VEMs.

This important characteristic can bring difficulties to correctly simulate the dynamic behavior of viscoelastic materials: Dispersions are inherent to experimental tests, consequently, the fitted parameters tend to be inaccurate lending to imprecisions in the computational modelling. In that way, the present work proposes a nondeterministic GHM method based approach which takes into account incertitudes in the VEM curve fitting procedure. The proposed methodology is validated with experimental results extracted from the literature and the results are considered as fair.

Similar results were achieved using also Anelastic Displacement Field (ADF) method [13] in instead of GHM, however, in order to avoid repetition, these results are not presented. 


\section{A summary of GHM Model}

The stress-strain relation on Laplace's domain as mentioned by Golla and Hughes [9] and McTavish and Hughes [10] (GHM) may be written as:

$$
\sigma(s)=\left[\mathrm{E}_{0}+\mathrm{h}(s)\right] \varepsilon(s)
$$

where $s$ is the Laplace operator, $\sigma(s)$ and $\varepsilon(s)$ are, respectively, the stress and strain on Laplace's domain, $\mathrm{E}_{0}$ is the elastic fraction of complex modulus and $\mathrm{h}(s)$ is the relaxation function. Function $\mathrm{h}(s)$ can be written using Biot's series with four terms (or two GHM terms):

$$
\mathrm{h}(s)=\alpha_{1} \frac{s^{2}+\beta_{1} s}{s^{2}+\beta_{1} s+\delta_{1}}+\alpha_{2} \frac{s^{2}+\beta_{2} s}{s^{2}+\beta_{2} s+\delta_{2}}
$$

where $\alpha_{i}, \beta_{i}$ and $\delta_{i}$ are materials constants and $\left(\alpha_{i}, \beta_{i}\right.$, $\left.\delta_{\mathrm{i}}\right) \geq 0$. Starting from the equation of motion in the Laplace domain:

$$
\left\{\mathrm{M} s^{2}+\mathrm{K}\right\} \mathrm{q}(s)=f(s)
$$

where $\mathrm{M}, \mathrm{K}$ and $\mathrm{f}(s)$ are respectively the mass, stiffness and external loading in the Laplace domain, where:

$$
\mathrm{K}=\left[\mathrm{E}_{0}+\sum \alpha_{i}\right] \mathrm{K}_{\mathrm{V}}
$$

where: $K_{v}$ is the rigidity fraction associated with geometrical characteristics of the model. The GHM model defines the equation of motion in the time domain as:

$$
\begin{aligned}
& {\left[\begin{array}{ccc}
\mathrm{M} & 0 & 0 \\
0 & \frac{\alpha_{1}}{\delta_{1}} \mathrm{~K}_{\mathrm{v}} & 0 \\
0 & 0 & \frac{\alpha_{2}}{\delta_{2}} \mathrm{~K}_{\mathrm{v}}
\end{array}\right]\left\{\begin{array}{c}
\ddot{\mathrm{q}} \\
\ddot{\mathrm{z}}_{1} \\
\ddot{\mathrm{z}_{2}}
\end{array}\right\}+\left[\begin{array}{ccc}
\mathrm{C} & 0 & 0 \\
0 & \frac{\alpha_{1} \beta_{1}}{\delta_{1}} \mathrm{~K}_{\mathrm{v}} & 0 \\
0 & 0 & \frac{\alpha_{2} \beta_{2}}{\delta_{2}} \mathrm{~K}_{\mathrm{v}}
\end{array}\right]\left\{\begin{array}{c}
\dot{\mathrm{q}} \\
\dot{\mathrm{z}}_{1} \\
\dot{\mathrm{z}}_{2}
\end{array}\right\}+} \\
& {\left[\begin{array}{ccc}
\mathrm{K}_{\mathrm{v}}\left(\mathrm{E}_{0}+\alpha_{1}+\alpha_{2}\right) & -\alpha_{1} \mathrm{~K}_{\mathrm{v}} & -\alpha_{2} \mathrm{~K}_{\mathrm{v}} \\
-\alpha_{1} \mathrm{~K}_{\mathrm{v}} & \mathrm{K}_{\mathrm{v}} & 0 \\
-\alpha_{2} \mathrm{~K}_{\mathrm{v}} & 0 & \mathrm{~K}_{\mathrm{v}}
\end{array}\right]\left\{\begin{array}{c}
\mathrm{q} \\
\mathrm{z}_{1} \\
\mathrm{z}_{2}
\end{array}\right\}=\left\{\begin{array}{c}
\mathrm{f}(t) \\
0 \\
0
\end{array}\right\}}
\end{aligned}
$$

where $z_{\mathrm{i}}$ is the auxiliary variable introduced into the problem, called dissipation variable. Generalizing Equation 5 for $n$ degrees of freedom, Equation 6 may be written as:

$$
\begin{aligned}
& {\left[\begin{array}{ccc}
\mathbf{M} & 0 & 0 \\
0 & \frac{\alpha_{1}}{\delta_{1}} \mathbf{I} & 0 \\
0 & 0 & \frac{\alpha_{2}}{\delta_{2}} \mathbf{I}
\end{array}\right]\left\{\begin{array}{l}
\ddot{\mathbf{q}} \\
\ddot{\mathbf{z}}_{1} \\
\ddot{\mathbf{z}}_{2}
\end{array}\right\}+\left[\begin{array}{ccc}
\mathbf{0} & \mathbf{0} & \mathbf{0} \\
\mathbf{0} & \frac{\alpha_{1} \beta_{1}}{\delta_{1}} \mathbf{I} & \mathbf{0} \\
\mathbf{0} & \mathbf{0} & \frac{\alpha_{2} \beta_{2}}{\delta_{2}} \mathbf{I}
\end{array}\right]\left\{\begin{array}{c}
\dot{\mathbf{q}} \\
\dot{\mathbf{z}}_{1} \\
\dot{\mathbf{z}}_{2}
\end{array}\right\}+} \\
& {\left[\begin{array}{ccc}
\mathbf{K}_{\mathbf{v}}\left(\mathrm{E}_{0}+\alpha_{1}+\alpha_{2}\right) & -\alpha_{1} \mathbf{R} & -\alpha_{2} \mathbf{R} \\
-\alpha_{1} \mathbf{R}^{\mathrm{T}} & \alpha_{1} \mathbf{I} & \mathbf{0} \\
-\alpha_{2} \mathbf{R}^{\mathrm{T}} & \mathbf{0} & \alpha_{2} \mathbf{I}
\end{array}\right]\left\{\begin{array}{c}
\mathbf{q} \\
\mathbf{z}_{1} \\
\mathbf{z}_{2}
\end{array}\right\}=\left\{\begin{array}{c}
\mathbf{f}(t) \\
\mathbf{0} \\
\mathbf{0}
\end{array}\right\}}
\end{aligned}
$$

where: $\mathbf{M}$ is the mass matrix of the corresponding elastic element,

$$
\mathbf{K}_{\mathbf{v}}=\mathbf{T}^{\mathrm{T}} \boldsymbol{\Lambda} \mathbf{T}
$$

and $\Lambda$ is a diagonal matrix consisting of the non-zero eigen-values of the stiffness matrix normalized with respect to the elastic modulus, E; $\mathbf{T}$ the matrix of vectors corresponding to the non-zero eigen-values of the matrix $(1 / E) \mathbf{K}_{\text {elastic }} ; \mathbf{R}=\mathbf{T} \Lambda^{1 / 2}$ and $\mathbf{z}_{\mathrm{i}}=\mathbf{R} \mathbf{z}_{\mathrm{i}}$.
As shown in Equations 6 and 7 the number of dissipative degrees of freedom associated with viscoelastic elements depends on the number of terms used in relaxation function and the number of rigid body motions [14]. It should be noted that the greater number of terms used to write the relaxation function more accurate the model will be.

\section{Experimental results used for validation}

In order to evaluate the proposed methodology, experimental data was taken from literature. Borges [15] performed a wide program of laboratory studies. From these laboratory studies, a set of two kinds of sandwich beams were selected to be used in the present paper:

- VS1 beam, with two elastic layers (base beam and clamped restraining layer) and one viscoelastic layer - see Figure 1a;

- VS1c beam, with two elastic layers (base beam and free restraining layer) and one viscoelastic layer - see Figure $2 b$.

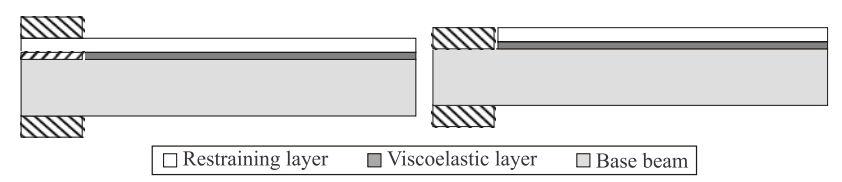

Figure 1. a - Schema of VS1 beam. b - Schema of VS1c beam.

All beams have rectangular cross section and 1140 mm length, working as the elastic base structure with $16,1 \mathrm{~mm}$ height; viscoelastic layers with $2,0 \mathrm{~mm}$ height; and elastic constraining layers with $3,17 \mathrm{~mm}$ height.

The elastic material was aluminium and the viscoelastic material used was VHB 4955. Mechanical properties of the materials are listed in Table 1.

Table 1. Mechanical properties of beam materials.

\begin{tabular}{|c|c|c|}
\hline Property & Aluminium & VHB4955 \\
\hline $\mathrm{E}(\mathrm{GPa})$ & 48.878 & - \\
\hline$v$ & 0.30 & 0.49 \\
\hline$\rho\left(\mathrm{kg} / \mathrm{m}^{3}\right)$ & 2690.0 & 795.0 \\
\hline
\end{tabular}

Five samples of each kind of tested beam were assayed. This strategy was applied in order to evaluate the experimental result dispersion. The samples were excited under the action of a hammer impact at $15 \mathrm{~cm}$ from cantilever and at the same section the transversal displacements were measured with LVDT sensors, which could register displacements without touching the structure.

\section{The proposed methodology}

The first step is to curve fit the experimental values of the frequency dependent Young Modulus, $E^{\prime}(\omega)$, and Loss Factor, $\eta(\omega)$, to the experimental data presented by 
Borges. In the case of the applied formulation GHM, they are given by Equations 8 and 9, respectively.

$$
\begin{gathered}
\mathrm{E}^{\prime}(\omega)=\mathrm{E}_{0}+\sum_{j=1}^{2} \alpha_{j} \frac{\omega^{2}\left(\omega^{2}-\delta_{j}+\beta_{j}^{2}\right)}{\left(\delta_{j}-\omega^{2}\right)^{2}+\beta_{j}^{2} \omega^{2}} \\
\eta(\omega)=\frac{1}{\mathrm{E} \prime(\omega)}+\sum_{j=1}^{2} \frac{\alpha_{j} \beta_{j} \delta_{j} \omega}{\left(\delta_{j}-\omega^{2}\right)^{2}+\beta_{j}^{2} \omega^{2}}
\end{gathered}
$$

In Equations 8 and 9, the set of parameters $E_{0}, \alpha_{j}, \beta_{j}$ and $\delta_{\mathrm{j}},(\mathrm{j}=1 \ldots 2)$ are unknown and, in the present work, they were curve fitted using Particle Swarm Optimization (PSO) algorithm $[16,12]$.

The adjustment for the best six set of parameters is presented in Figures 2 and 3.

Figure 2. The best six $E^{\prime}(\omega)$ adjustments.

Figure 3. The best six $\eta(\omega)$ adjustments.

Defining the best adjustment is not an easy task, as it changes depending on the adopted evaluation criteria. In that way and considering the incertitudes of the experimental data, all these six adjustments will be used in the computational modelling. Table 2 presents each set of parameters achieved during the curve fitting process.

The next step is to simulate the experimental tests. In order to numerically simulate the dynamical behavior of the beams, the structures were discretized with CST meshes as the one shown in Figure 4. These meshes had 98,084 physical dof and 54,720 dissipation dof, 152,804 in total for VS1 and VS1c beams, respectively.

Table 2. Set of adjusted parameters.

\begin{tabular}{|c|c|c|c|c|c|c|c|}
\hline $\begin{array}{c}\text { Adjust- } \\
\text { ment }\end{array}$ & $\begin{array}{c}\mathbf{E}_{\mathbf{0}} \\
\left(\mathrm{x} 10^{5}\right)\end{array}$ & $\begin{array}{c}\boldsymbol{\alpha}_{\mathbf{1}} \\
\left(\mathrm{x} 10^{6}\right)\end{array}$ & $\begin{array}{c}\boldsymbol{\alpha}_{\mathbf{2}} \\
\left(\mathrm{x} 10^{6}\right)\end{array}$ & $\begin{array}{c}\boldsymbol{\beta}_{\mathbf{1}} \\
\left(\mathrm{x} 10^{7}\right)\end{array}$ & $\begin{array}{c}\boldsymbol{\beta}_{\mathbf{2}} \\
\left(\mathrm{x} 10^{7}\right)\end{array}$ & $\begin{array}{c}\boldsymbol{\delta}_{1} \\
\left(\mathrm{x} 10^{9}\right)\end{array}$ & $\begin{array}{c}\boldsymbol{\delta}_{\mathbf{2}} \\
\left(\mathrm{x} 10^{9}\right)\end{array}$ \\
\hline 1 & 4.458 & 5.880 & 1.254 & 0.399 & 7.533 & 4.485 & 4.115 \\
\hline 2 & 6.047 & 1.891 & 7.966 & 1.529 & 0.553 & 0.979 & 6.952 \\
\hline 3 & 2.843 & 0.771 & 3.803 & 8.435 & 0.562 & 4.048 & 5.477 \\
\hline 4 & 3.734 & 7.578 & 0.940 & 0.592 & 8.964 & 6.754 & 4.242 \\
\hline 5 & 4.664 & 1.151 & 9.000 & 1.069 & 0.246 & 0.499 & 2.625 \\
\hline 6 & 2.483 & 0.600 & 5.034 & 5.716 & 0.483 & 2.586 & 5.346 \\
\hline
\end{tabular}

Figure 4. Viscoelastic sandwich cantilever beam structural Finite Element discretization.

The models were simulated under the action of a hammer impact at $15 \mathrm{~cm}$ from cantilever and, at same point, it was observed the transversal displacement along the time. With the models given by Equations 6, the time response of the beams could be obtained. Natural frequencies and damping rates were extracted by means of an automatic Stochastic Subspace Identification algorithm as proposed by Cabboi et al. [17].

The numerical simulation was performed using Monte Carlo Simulations, where there are generated $N$ random samples and, at the end of simulations, the results obtained with each sample are condensed. In this case each sample has a unique random spatial distribution of the six viscoelastic fitted curves. A typical example of this spatial distribution is illustrated in Figure 5. In this figure are shown both elastic elements (in gray) as well as the viscoelastic fitted curves (six colors, one for each fitted curve). In this work were performed 50 independent runs, one for each sample generated.

Figure 5. Typical spatial distribution of the viscoelastic fitted curves for VS1 beam.

At the end of the simulations the dispersion results for the first three vibration modes were obtained, as can be seen in Table 3 , in terms of damping ratios $\left(\xi_{1}, \xi_{2}\right.$ and $\left.\xi_{3}\right)$, and in Table 4 , in terms of natural frequencies $\left(f_{1}, f_{2}\right.$ and $f_{3}$ ). These Tables present the experimental results, the dispersions obtained with the nondeterministic model, the mean values and standard-deviations, and the results obtained through a deterministic analysis using each fitted curve separately.

Table 3. Numerical and experimental damping ratios of the

\begin{tabular}{|c|c|c|c|c|c|}
\hline \multirow{2}{*}{ Beam } & \multirow{2}{*}{\multicolumn{2}{|c|}{ Model }} & \multicolumn{3}{|c|}{ Damping Ratios (\%) } \\
\hline & & & $\xi_{1}$ & $\xi_{2}$ & $\xi_{3}$ \\
\hline \multirow{8}{*}{ VS1 } & \multicolumn{2}{|c|}{ Experimental } & $\begin{array}{c}4.44 \\
\pm 0.01\end{array}$ & $\begin{array}{c}4.32 \\
\pm 0.05\end{array}$ & $\begin{array}{c}3.28 \\
\pm 0.06 \\
\end{array}$ \\
\hline & \multicolumn{2}{|c|}{ Nondeterministic } & $\begin{array}{c}4.52 \\
\pm 0.21\end{array}$ & $\begin{array}{c}4.09 \\
\pm 0.75\end{array}$ & $\begin{array}{c}1.93 \\
\pm 0.29\end{array}$ \\
\hline & \multirow{6}{*}{ Deterministic } & $\# 1$ & 5.24 & 5.74 & 1.54 \\
\hline & & \#2 & 4.54 & 5.90 & 2.441 \\
\hline & & $\# 3$ & 4.35 & 3.23 & 1.71 \\
\hline & & $\# 4$ & 4.64 & 6.35 & 1.90 \\
\hline & & $\# 5$ & 4.53 & 4.06 & 2.02 \\
\hline & & $\# 6$ & 4.23 & 4.64 & 1.45 \\
\hline \multirow{8}{*}{ VS1c } & \multicolumn{2}{|c|}{ Experimental } & $\begin{array}{c}2.74 \\
\pm 0.01\end{array}$ & $\begin{array}{c}4.80 \\
\pm 0.10\end{array}$ & $\begin{array}{c}4.44 \\
\pm 0.04\end{array}$ \\
\hline & \multicolumn{2}{|c|}{ Nondeterministic } & $\begin{array}{c}1.80 \\
\pm 0.03\end{array}$ & $\begin{array}{c}3.43 \\
\pm 0.15\end{array}$ & $\begin{array}{c}1.90 \\
\pm 0.17\end{array}$ \\
\hline & \multirow{6}{*}{ Deterministic } & $\# 1$ & 1.81 & 4.08 & 1.82 \\
\hline & & $\# 2$ & 2.30 & 5.90 & 1.82 \\
\hline & & $\# 3$ & 1.37 & 4.63 & 1.81 \\
\hline & & $\# 4$ & 1.78 & 6.18 & 2.11 \\
\hline & & $\# 5$ & 2.01 & 3.78 & 1.97 \\
\hline & & \#6 & 1.39 & 4.26 & 1.49 \\
\hline
\end{tabular}
analysed beams.

Table 4. Numerical and experimental natural frequencies of the analysed beams.

\begin{tabular}{|c|c|c|c|c|c|}
\hline \multirow{3}{*}{ Beam } & \multirow{2}{*}{ Model } & \multicolumn{3}{|c|}{ Natural frequency $(\mathbf{H z})$} \\
\cline { 3 - 6 } & \multicolumn{2}{|c|}{} & $\boldsymbol{f}_{\mathbf{1}}$ & $\boldsymbol{f}_{\mathbf{2}}$ & $\boldsymbol{f}_{\mathbf{3}}$ \\
\hline \multirow{3}{*}{ VS1 } & \multirow{2}{*}{ Experimental } & 11.03 & 61.76 & 168.08 \\
& \multirow{2}{*}{ Nondeterministic } & 10.02 & \pm 0.14 & \pm 0.23 \\
\cline { 3 - 6 } & \multirow{2}{*}{} & \pm 0.02 & 62.50 & 159.35 \\
& \multirow{2}{*}{ Deterministic } & $\# 1$ & 10.95 & 62.63 & 159.23 \\
\cline { 3 - 6 } & & $\# 2$ & 11.19 & 63.26 & 160.68 \\
\hline
\end{tabular}




\begin{tabular}{|c|c|c|c|c|c|}
\hline & & $\# 3$ & 10.62 & 61.71 & 157.60 \\
\hline & & \#4 & 10.85 & 62.46 & 159.82 \\
\hline & & $\# 5$ & 11.02 & 63.30 & 161.12 \\
\hline & & \#6 & 10.58 & 61.60 & 157.96 \\
\hline \multirow{8}{*}{ VS1c } & \multicolumn{2}{|c|}{ Experimental } & $\begin{array}{c}9.82 \\
\pm 0.00\end{array}$ & $\begin{array}{l}63.70 \\
\pm 0.04\end{array}$ & $\begin{array}{c}174.05 \\
\pm 0.35\end{array}$ \\
\hline & \multicolumn{2}{|c|}{ Nondeterministic } & $\begin{array}{c}9.73 \\
\pm 0.01\end{array}$ & $\begin{array}{l}62.28 \\
\pm 0.05\end{array}$ & $\begin{array}{c}158.96 \\
\pm 0.13\end{array}$ \\
\hline & \multirow{6}{*}{ Deterministic } & \#1 & 9.75 & 62.45 & 158.70 \\
\hline & & \#2 & 9.84 & 63.47 & 160.13 \\
\hline & & \#3 & 9.66 & 61.35 & 157.09 \\
\hline & & $\# 4$ & 9.72 & 62.39 & 159.27 \\
\hline & & $\# 5$ & 9.77 & 63.14 & 16.53 \\
\hline & & \#6 & 9.65 & 61.60 & 157.47 \\
\hline
\end{tabular}

\section{Discussions and Conclusions}

It was presented and implemented a nondeterministic model based on the Monte Carlo method for viscoelastic materials via GHM technique that simulates the uncertainty of complex modulus in a simplified manner. The results obtained with this model were compared with experimental data and deterministic models.

Regarding the accuracy of numerical models, it can be seen in Table 5 that in general the results obtained with the nondeterministic models are better than those obtained in a deterministic way. It is noteworthy that the performance of these models, deterministic and nondeterministic, are strongly depend on the fitted parameters considered. Once the nondeterministic model uses several adjustments simultaneously, the risk of improperly evaluate the dynamic behavior of a structure, and, consequently, to obtain inconsistent results is smaller. In the case of beam VS1c the numerical results are close to the first two experimental frequencies "by luck". This may be due to the complex modulus adjustment exhibits a better correlation with the experimental data at this frequency range.

In general, the non-deterministic model showed the best results, being promising. Nevertheless, the results can be significantly improved since:

- The adjustments were distributed randomly, this distribution did not considered the probability distribution function of experimental characterization data;

- The set of adjusted curves was obtained trying to approximate the average value of characterization data. It is believed that the model performance can be improved generating sets considering also the dispersion of characterization results;

- The simulations were performed without any criteria for sample selection.

In addressing these points, one can say that the points to be improved in the model are: the sampling methodology and the distribution of the adjusted curves over the viscoelastic domain. Regarding the sampling Janssen [18] presents a series of sampling Monte Carlo simulations to substantially reduce the number of simulations required to achieve good results. Regarding the distribution of adjustments, some authors $[19,20]$ applied the Karhunen-Loève expansion to perform the discretization of the random field successfully.

Table 5. Errors for numerical natural frequencies identified of the analysed beams.

\begin{tabular}{|c|c|c|c|c|c|c|c|c|}
\hline \multirow{2}{*}{ Beam } & \multirow{2}{*}{\multicolumn{2}{|c|}{ Model }} & \multicolumn{3}{|c|}{ Error on Natural frequency $(\%)$} & \multicolumn{3}{|c|}{ Error on Damping ratios (\%) } \\
\hline & & & $f_{1}$ & $f_{2}$ & $f_{3}$ & $\xi_{1}$ & $\xi_{2}$ & $\xi_{3}$ \\
\hline \multirow{7}{*}{ VS1 } & \multicolumn{2}{|c|}{ Nondeterministic } & -1.45 & 1.20 & -.519 & 1.80 & -5.32 & -41.46 \\
\hline & \multirow{6}{*}{ Deterministic } & $\# 1$ & -0.73 & 1.41 & -5.27 & 18.02 & 32.87 & -53.05 \\
\hline & & $\# 2$ & 1.45 & 2.43 & -4.40 & 2.25 & 36.57 & -26.52 \\
\hline & & $\# 3$ & -3.72 & -0.08 & -6.24 & -2.03 & -25.23 & -47.87 \\
\hline & & $\# 4$ & -1.63 & 1.13 & -4.91 & 4.50 & -15.51 & -42.07 \\
\hline & & \#5 & -0.09 & 2.49 & -4.14 & 32.03 & -6.02 & -38.41 \\
\hline & & \#6 & -4.08 & -0.26 & --6.02 & -4.73 & 7.41 & -55.79 \\
\hline \multirow{7}{*}{ VS1c } & \multicolumn{2}{|c|}{ Nondeterministic } & -0.92 & -2.34 & -8.67 & -34.31 & -28.54 & -57.21 \\
\hline & \multirow{6}{*}{ Deterministic } & $\# 1$ & -0.71 & -2.07 & -8.82 & -33.94 & -15.00 & -59.01 \\
\hline & & $\# 2$ & 0.20 & -0.47 & -8.00 & -16.06 & 22.92 & -59.01 \\
\hline & & \#3 & -1.63 & -3.79 & -9.74 & -50.00 & -3.54 & -59.23 \\
\hline & & $\# 4$ & -1.02 & -2.16 & -8.49 & -35.04 & 28.57 & -52.48 \\
\hline & & $\# 5$ & -0.51 & -0.99 & -7.77 & -26.64 & -21.25 & -55.63 \\
\hline & & \#6 & -1.73 & -3.40 & -9.53 & -49.27 & -11.25 & -66.44 \\
\hline
\end{tabular}




\section{References}

1. R.C. Battista, E.F. Santos, R. Vasconcelos, M.S. Pfeil, In Stability and Ductility of Steel Structures (2010)

2. J. Kim, J. Ryu, C. Lan, Eng. Struc. 28, 183-195 (2006)

3. E. Moliner, P. Museros, M.D. Martnez-Rodrigo, Eng. Struc. 40, 519-528 (2012)

4. I. Saidi, E.F. Gad, J.L. Wilson, N. Haritos, Eng. Struc. 33, 3317-3328 (2011)

5. M.I. Friswell, J.K. Dutt, S. Adhikari, A.W. Lees, Int. Jour. Mech. Sc. 52, 1319-1324 (2010)

6. H. Roy, J.K. Dutt, P.K. Datta, Int. Jour. Mech. Sc. 50, 845-853 (2012)

7. G. Wang, S. Veeramani, N.M. Wereley, Jour. Vib. Acous. 112, 305-312 (2000).

8. Y. Wang, D.J. Inman, Jour. Sound and Vib. 332, 6177-6191 (2013)

9. D.F. Golla, P.C. Hughes, Jour. App. Mech. 52, $897-$ 906 (1985)

10. D.J. McTavish, P.C. Hughes, Jour. Vib. Acou. 115, 103-113 (1993)

11. F.S. Barbosa, M.C.R. Farage, Jour. Sound and Vib. 317, 91-111 (2008)

12. W.N. Felippe-Filho, F.S. Barbosa, Mec. Comp. 1557-1570 (2014)

13. G.A. Lesieutre, E. Bianchini, Jour. Vib. Acou. 117, 424-430 (1993)

14. F.S. Barbosa, Modelagem computacional de estruturas com camadas viscoelásticas amortecedoras. Phd-thesis, COPPE/UFRJ (2000)

15. F.C. Borges, Análise do comportamento dinâmico de vigas sanduiche com múltiplas camadas. M.Sc.thesis, COPPE/UFRJ (2010)

16. J. Kennedy, R.C. Eberhart, In International conference on neural networks (1995)

17. A. Cabboi, F. Magalhaes, genile C., A. Cunha, In 6th Conference on Smart Structures and Materials (2013)

18. H. Janssen, Rel. Engrg. Sys. Saf. 109, 213-132 (2013)

19. S. Shang, G.J. Yun, Fin. Elem. Anal. Design. 64, 6578 (2013)

20. W. Betz, I. Papaioannou, D. Straub, Comput. Meth. Appl. Mech. Engrg. 271, 109-129 (2014) 